\title{
Finite volume effects for the pion mass at two loops
}

\author{
Gilberto Colangelo and Christoph Haefeli \\ Institut für Theoretische Physik, Universität Bern \\ Sidlerstr. 5, 3012 Bern, Switzerland
}

\begin{abstract}
We evaluate the pion mass in finite volume to two loops within Chiral Perturbation Theory. The results are compared with a recently proposed extension of the asymptotic formula of Lüscher. We find that contributions, which were neglected in the latter, are numerically very small at the two-loop level and conclude that for $M_{\pi} L \gtrsim 2, L \geq 2 \mathrm{fm}$ the finite volume effects in the meson sector are analytically well under control.
\end{abstract}

\section{Introduction}

Numerical simulations in lattice QCD are bound to rather small volumes. When one determines the hadron spectrum and other low energy observables one has to understand and properly account for the volume dependence in order to correctly interpret the numerical data. Analytical methods which allow one to predict the size of the finite volume effects are particularly useful in this respect. In the case of hadron masses, there are two different methods to analytically evaluate the finite volume effects: the asymptotic formula derived by Lüscher [1], and chiral perturbation theory (ChPT) in finite volume [2, 3, 4, Lüscher's formula relates the volume dependence of the mass of a hadron to an integral over the $\pi$-hadron forward scattering amplitude. Knowledge of the latter scattering amplitude immediately translates into an estimate of finite volume effects for the hadron mass. Since the integral is dominated by the low-energy region, one can rely on the chiral representation of the scattering amplitude in order to numerically evaluate the integral. In this manner one makes use of ChPT only in infinite volume and obtains an estimate only of the leading exponential term in the finite volume dependence, the term of the order $\exp \left(-M_{\pi} L\right)$, where $L$ is the box size. Alternatively one can 
perform the calculation of the hadron mass in ChPT in finite volume and obtains an estimate also of the terms which are exponentially subleading.

A detailed study and comparison of the two approaches has been performed in [5, 6] for the case of the pion mass. This case is particularly interesting both because it is a simple hadron to be studied on the lattice and because the $\pi \pi$ scattering amplitude, which is needed in the Lüscher formula, is now known to next-to-next-to-leading order [7, 8, 9, 10] in the chiral expansion. It was therefore possible to study numerically how well the leading exponential term dominates the series, and how fast the chiral expansion (of the leading exponential term) converges. The somewhat surprising result was that the leading term in both series receives large corrections both from subleading exponentials as well as from the next-to-leading order in the chiral expansion. The next-to-next-to-leading order chiral correction, on the other hand, was found to be rather small, which indicates that - at this level - the chiral series has started converging well.

The fact that the Lüscher formula appeared to give the numerically dominating term only for volumes so large that the finite volume correction itself has become negligible appeared to be more worrisome. At first sight this might have lead one to conclude that in cases of practical interest one could not rely on this extremely convenient formula in order to evaluate reliably the finite volume effects. We believe, however, that such a negative conclusion is unjustified and have proposed a resummation of the Lüscher formula [11,12] which retains all the convenience of the original one but does not suffer from the same large corrections. This resummation can be understood in simple terms: Lüscher has shown that the leading exponential correction comes from a radiative correction in which the emitted virtual light particle (the pion, in QCD) goes around the world once (thanks to the periodic boundary conditions) before being reabsorbed. The resummed formula takes into account all other possible ways which the pion has to go around the world (go along the diagonal, or go around more than once, etc.).

As was shown in [12] this resummed formula exactly reproduces the one-loop ChPT calculation of the pion mass in finite volume if one inserts in the integral the leading chiral representation of the $\pi \pi$ scattering amplitude. Inserting the nextto-leading chiral representation of the scattering amplitude one reproduces the full two-loop calculation of the pion mass in ChPT in finite volume up to corrections of order $\exp (-\bar{M} L)$ with $\bar{M} \geq(\sqrt{3}+1) / \sqrt{2} M_{\pi}$. Despite this improvement of the algebraic accuracy of the resummed formula with respect to the Lüscher's one, it is essential to show that the resummation is numerically effective and that the corrections to it are small. In order to make this check we have now performed a full two loop calculation of the pion mass in finite volume in ChPT. As we 
will demonstrate in this article, the corrections which are not captured by the resummed asymptotic formula are negligibly small for $M_{\pi} L \gtrsim 2$. The results of the present two-loop calculation were anticipated in [13, 14. Here, we give further details about the calculation and the results.

One of the reasons for performing this further investigation of finite volume effects for hadron masses is that one may view Lüscher's formula (or its resummed version) as a way to determine on the lattice a scattering amplitude, cf. [12. While it is true that the scattering amplitude is seen here in an exponentially suppressed effect, it is also true that the direct calculation of a scattering amplitude on the lattice is much more difficult than that of a mass. This indirect method to extract a scattering amplitude may in some cases turn out to be more practical, and we find it worthwhile to discuss its theoretical feasibility. Indeed if the part of the finite volume corrections which is related to the scattering amplitude is not strongly dominating with respect to the rest, this is not a viable method to determine the scattering amplitude. The conclusion reached in this paper is therefore encouraging in this respect and can be used to estimate in which region of the $\left(M_{\pi}, L\right)$ plane the finite volume correction to a hadron mass is given to a good approximation by the integral containing the scattering amplitude.

The two-loop calculation is interesting in its own right, also from a technical viewpoint. To date, a number of finite volume calculations have been performed at one-loop order [15, 16, 17, 18, 19, 20, 21, but as far as we know the only finite volume two-loop calculation which has been performed until now is for the quark condensate [22], which is a much simpler calculation. In the related context of finite temperature field theory there are a few examples of calculations beyond one loop [23, 24].

We wish to briefly mention related work. Most notably, the asymptotic formula may also be applied to the nucleon mass [25], see Koma and Koma [26] as well as [14] for recent work. Braun, Pirner and Klein have evaluated the volume dependence of the pion mass based on a quark-meson model [27]. In the framework of a lattice regularised ChPT finite volume effects have been addressed by Borasoy et al. in Ref. [28].

The outline of the article is as follows. In sect. 2 we set the notation and remind of the basic assumptions for an application of ChPT in finite volume. Sect. 3 is devoted to outline the calculation and state the main results. In sect. 4 we show the explicit expressions which have been used for the numerical analysis in sect. 5 . We conclude with a summary. 


\section{Preliminaries}

In this section we shall set the notation and the basic definitions for the two-loop calculation.

\subsection{ChPT in finite and in infinite volume}

Chiral Perturbation Theory (ChPT) is the effective theory for QCD at low energies. If we first restrict ourselves to the infinite volume case, the effective Lagrangian of QCD for two light flavours at low energies consists of an infinite number of terms 7.

$$
\mathcal{L}_{\text {eff }}=\mathcal{L}_{2}+\mathcal{L}_{4}+\mathcal{L}_{6}+\ldots
$$

As we wish to calculate the pion mass, an on-shell quantity, external fields can be dropped in $\mathcal{L}_{\text {eff }}$. We work in the isospin symmetry limit $m_{u}=m_{d}$ in Euclidean space-time, and for the choice of the pion fields we use the non-linear sigma model parameterization. We have

$$
\mathcal{L}_{2}=\frac{F^{2}}{4}\left\langle u_{\mu} u_{\mu}-\chi_{+}\right\rangle
$$

with

$$
\begin{aligned}
& U=\sigma+i \frac{\phi}{F} \quad, \quad \sigma^{2}+\frac{\phi^{2}}{F^{2}}=\mathbb{1}, \quad \phi=\left(\begin{array}{cc}
\pi^{0} & \sqrt{2} \pi^{+} \\
\sqrt{2} \pi^{-} & -\pi^{0}
\end{array}\right)=\phi^{i} \tau^{i} \\
& u_{\mu}=i u^{\dagger} \partial_{\mu} U u^{\dagger}=-i u \partial_{\mu} U^{\dagger} u=u_{\mu}^{\dagger}, \quad \chi_{+}=u^{\dagger} \chi u^{\dagger}+u \chi^{\dagger} u \\
& \chi=2 B \hat{m} \mathbb{1} \quad, \quad \hat{m}=\frac{1}{2}\left(m_{u}+m_{d}\right)
\end{aligned}
$$

with $u^{2}=U$. The symbol $\langle A\rangle$ denotes the trace of the two-by-two matrix $A$. The Lagrangian $\mathcal{L}_{4}$ can be written as [7],

$$
\mathcal{L}_{4}=\sum_{i=1}^{4} \ell_{i} P_{i}+\ldots
$$

where

$$
P_{1}=-\frac{1}{4}\left\langle u_{\mu} u_{\mu}\right\rangle^{2}, P_{2}=-\frac{1}{4}\left\langle u_{\mu} u_{\nu}\right\rangle^{2}, P_{3}=-\frac{1}{16}\left\langle\chi_{+}\right\rangle^{2}, P_{4}=\frac{i}{4}\left\langle u_{\mu} \chi_{-\mu}\right\rangle
$$

with

$$
\chi_{-\mu}=u^{\dagger} \partial_{\mu} \chi u^{\dagger}-u \partial_{\mu} \chi^{\dagger} u
$$


The ellipsis in eq. (4) denotes terms that do not contribute to the pion mass. The low energy constants $\ell_{i}$ are divergent and remove the ultraviolet divergences generated by one-loop graphs from $\mathcal{L}_{2}$.

The complete effective Lagrangian $\mathcal{L}_{6}$ with its divergence structure at $d=4$ has been constructed in [29, 30]. As will be discussed in sect. 3] terms from $\mathcal{L}_{6}$ merely renormalize the pion mass in infinite volume and do not contribute to finite volume corrections which we are interested in. Thus, we refrain from showing it here. Given the effective Lagrangian and the parameterization for the pion fields, it is straightforward to calculate the pion mass to two-loops. We refer to [9], where one also finds a detailed discussion of the renormalization procedure [two-loop diagrams in ChPT are discussed in [31]].

The effective framework is still appropriate, when the system is enclosed by a large box of size $V=L^{3}$. We refer to the literature for the foundations [2, 3, 4] and a recent review [11]. Here, we only recall a few fundamental results which guided the present calculation: the volume has to be large enough, such that ChPT can give reliable results, $2 F_{\pi} L \gg 1$. The value of the parameter $M_{\pi} L$ determines the power counting for the perturbative calculation: if $M_{\pi} L \gg 1$ one is in the " $p$ regime" in which $1 / L$ counts as a small quantity of order $M_{\pi}$. If $M_{\pi} L \lesssim 1$ one is in the " $\epsilon$-regime" and $1 / L^{2}$ is a quantity of order $M_{\pi}$. In both cases the effective Lagrangian is the same as in the infinite volume. In this article we only consider the " $p$-regime", where the system is distorted mildly and the only change brought about by the finite volume is a modification of the pion propagator due to the periodic boundary conditions of the pion fields ${ }^{1}$

$$
G\left(x^{0}, \mathbf{x}\right)=\sum_{\mathbf{n} \in \mathbb{Z}^{3}} G_{0}\left(x^{0}, \mathbf{x}+\mathbf{n} L\right)
$$

with $G_{0}(x)$ the propagator in infinite volume.

\subsection{Basic definitions}

In Euclidean space-time the propagator is defined through the connected correlation function

$$
G(x) \delta^{a b}=\left\langle\phi^{a}(x) \phi^{b}(0)\right\rangle_{L},
$$

where $a, b$ stand for isospin indices of the pion fields and the subscript $L$ in the correlation function denotes that it is evaluated in finite volume.

\footnotetext{
${ }^{1}$ Throughout we denote by the volume the three-dimensional volume $V=L^{3}$, whereas the time direction is not compactified.
} 
We also have

$$
\begin{aligned}
\left\langle\phi^{1}(x) \phi^{1}(0)\right\rangle_{L} & =L^{-3} \sum_{\mathbf{p}} \int \frac{d p^{0}}{2 \pi} e^{i p x} G\left(p^{0}, \mathbf{p}\right), \\
G\left(p^{0}, \mathbf{p}\right)^{-1} & =M^{2}+p^{2}-\Sigma_{L}\left(p^{0}, \mathbf{p}\right),
\end{aligned}
$$

where the momenta $\mathbf{p}$ can only have discrete values

$$
\mathbf{p}=\frac{2 \pi}{L} \mathbf{n}, \quad \mathbf{n} \in \mathbb{Z}^{3},
$$

and $M^{2}=2 B \hat{m}$ is the tree-level pion mass in infinite volume. The pion mass in finite volume $M_{\pi L}$ is now defined by the pole equation

$$
G\left(\hat{p}_{L}\right)^{-1}=0, \quad \text { for } \quad \hat{p}_{L}=\left(i M_{\pi L}, \mathbf{0}\right) .
$$

\section{Outline of calculation and statement of results}

For a large volume, the finite size effects are expected to be small, such that the pole equation can be solved perturbatively. We outline the calculation and proceed with the main results. More details about the calculation are relegated to later sections or the appendix.

\subsection{One-loop result}

Since the effective Lagrangian remains unchanged when going to the finite volume, we can immediately write down the Feynman diagrams which contribute to the self-energy at two-loops, see fig. 1 the only difference with respect to an infinite volume calculation is that the propagators need to be periodified, cf. eq.(17). At leading order, the graphs $1(a)$ and $1(e)$ need to be evaluated and the self-energy admits the form

$$
\Sigma_{L}\left(p^{0}, \mathbf{p}\right)=\frac{1}{F_{\pi}^{2}} G(0)\left(-\frac{3}{2} M_{\pi}^{2}-p^{2}\right)-2 \ell_{3} \frac{M_{\pi}^{4}}{F_{\pi}^{2}}+\mathcal{O}\left(\frac{1}{F_{\pi}^{4}}\right)
$$

with $G(0)$ the value of the finite volume propagator at the origin. It contains a logarithmic divergence due to the contribution from the term $\mathbf{n}=\mathbf{0}$ in eq.(17). In dimensional regularization,

$$
G_{0}(0)=\frac{1}{(2 \pi)^{d}} \int d^{d} p \frac{1}{p^{2}+M^{2}}=\frac{\Gamma(1-d / 2)}{(4 \pi)^{d / 2}} M^{d-2} .
$$




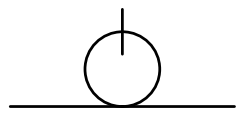

(a)

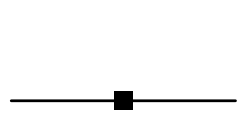

(e )

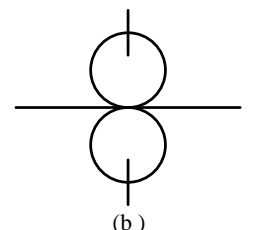

(b)

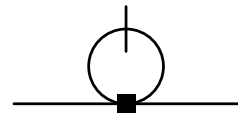

(f)

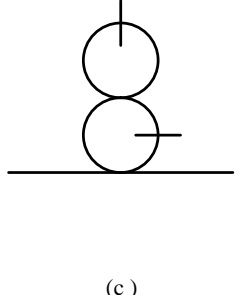

(c)

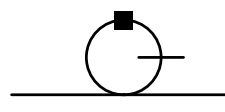

$(\mathrm{g})$

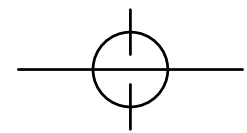

(d)

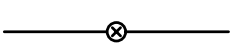

(h)

Figure 1: Self-energy graphs to two-loops in ChPT. A spline corresponds to a periodified propagator, whereas those without correspond to an infinite volume propagator, cf. eq.(17). Normal vertices come from $\mathcal{L}_{2}$, squared vertices from $\mathcal{L}_{4}$ and the circle-crossed from $\mathcal{L}_{6}$.

The remaining terms with $\mathbf{n} \neq \mathbf{0}$ are finite and may be expressed in terms of a kinematical function ${ }^{2} g_{1}\left(M^{2}, 0, L\right)$,

$$
\begin{aligned}
G(0) & =G_{0}(0)+g_{1}\left(M^{2}, 0, L\right) \\
g_{1}\left(M^{2}, 0, L\right) & =\int_{0}^{\infty} \frac{d \tau}{(4 \pi \tau)^{d / 2}} e^{-\tau M^{2}} \sum_{\mathbf{n} \neq \mathbf{0}} \exp \left(-\frac{\mathbf{n}^{2} L^{2}}{4 \tau}\right)
\end{aligned}
$$

For a derivation of eq.(14), we refer to [32. In app. @ we provide a different derivation, based on a contour integration analysis. The pole of the Gamma function in eq. (13) in four dimensions is absorbed in a renormalization of the low-energy constant $\ell_{3}$. One readily verifies that inserting eq.(14) into eq.(12) yields for the leading finite volume shift [2]

$$
M_{\pi L}=M_{\pi}\left[1+\frac{1}{4 F_{\pi}^{2}} g_{1}\left(M_{\pi}^{2}, 0, L\right)+\mathcal{O}\left(\frac{1}{F_{\pi}^{4}}\right)\right] .
$$

The separation of the cut-off and the volume-dependence is as expected: finite volume corrections do not generate new uv-divergences. At leading order the finite volume corrections could be isolated immediately. This will not be the case at the two-loop level. The graph $(d)$ in fig. 11 does not factorize in pure one-loop integrals and further steps need to be performed. We refer to app. @ for further details.

\footnotetext{
${ }^{2}$ The second argument of $g_{1}$ is the temperature, which we keep zero. Notation as in Ref.[2]
} 


\subsection{Minimal set of periodified propagators}

In the evaluation of the Feynman diagrams, it is mandatory to make use of the following result. In order to evaluate a graph consisting of $L$ loops, one needs to consider only a certain set of $L$ propagators as the periodified, finite-volume ones the others can be taken as infinite-volume propagators. In the following we briefly discuss this statement (see also p.18ff in Ref.[1]). Consider an arbitrary self-energy graph with $L$ loops, $I$ internal lines $\ell$ and $V$ vertices. Since the number of loops is the number of independent integrations over momenta, we have

$$
L=I-V+1 \text {. }
$$

For every line $\ell$ the propagator is an infinite sum of terms characterized by an integer vector $\mathbf{n}(\ell)$. The loop graph itself is an infinite sum of terms which can be identified by a set of integer vectors $\{\mathbf{n}(\ell)\}$ assigned to all the internal lines. As remarked by Lüscher this structure can be viewed as a gauge field on the self-energy graph. Gauge transformations are defined $\mathrm{as}^{3}$

$$
\mathbf{n}^{\prime}(\ell)=\mathbf{n}(\ell)+\mathbf{\Lambda}(f(\ell))-\mathbf{\Lambda}(i(\ell))
$$

where $\boldsymbol{\Lambda}(v)$ is some field of integer vectors and $i(\ell)(f(\ell))$ is the initial (final) vertex of the line $\ell$. One verifies immediately that two contributions of a Feynman graph which differ only by a gauge transformation yield the same mathematical expression. In the calculation of the loop graph what matters is the sum over representatives of gauge equivalence classes. A convenient representative can be found, e.g., by adjusting $\boldsymbol{\Lambda}(i(\ell))$ and $\boldsymbol{\Lambda}(f(\ell))$ iteratively, such that $\mathbf{n}(\ell)=0$ for as many internal lines $\ell$ as possible. This can be achieved for $V-1$ lines. Therefore, there remain $I-V+1$ internal lines where the periodified propagator has to be inserted - which coincides with the number of loops of the graph. This shall be our minimal set of periodified propagators. In fig. 1 we have attached a spline to a periodified propagator, whereas lines without a spline correspond to an infinite volume propagator.

\subsection{Two-loop result}

It is convenient to split the sum over the equivalence classes into three parts ${ }^{4}$ :

$$
\begin{array}{ll}
\Sigma^{(0)}: & \mathbf{n}(\ell)=0 \forall \ell \text { (pure gauge), } \\
\Sigma^{(1)}: & \mathbf{n}(\ell)=0 \forall \ell \text { except for one line } \bar{\ell} \text { (simple gauge), } \\
\Sigma^{(2):} & \mathbf{n}(\ell)=0 \forall \ell \text { except for two lines } \bar{\ell}_{1}, \bar{\ell}_{2} .
\end{array}
$$

\footnotetext{
${ }^{3}$ We borrow the notation from Ref. [1].

${ }^{4}$ Note the slight difference in the definition of simple fields with respect to [1. We do not require $|\mathbf{n}(\ell)|=1$.
} 
The pion mass in finite volume to two-loops then admits the form

$$
\begin{aligned}
M_{\pi L}^{2} & =M_{\pi}^{2}-\Sigma^{(1)}-\Sigma^{(2)} \\
M_{\pi}^{2} & =M^{2}-\Sigma^{(0)}
\end{aligned}
$$

where, using $\lambda_{\pi}=M_{\pi} L$, we get

$$
\begin{aligned}
\Sigma^{(1)} & =I_{p}+I_{c}+\mathcal{O}\left(\xi^{3}\right) \\
I_{p} & =\frac{M_{\pi}^{2}}{16 \pi^{2} \lambda_{\pi}} \sum_{n=1}^{\infty} \frac{m(n)}{\sqrt{n}} \int_{-\infty}^{\infty} d y \mathcal{F}_{\pi}(\mathrm{i} y) e^{-\sqrt{n\left(1+y^{2}\right)} \lambda_{\pi}} \\
I_{c} & =-\frac{\mathrm{i} M_{\pi}^{2}}{32 \pi^{3} \lambda_{\pi}} \sum_{n=1}^{\infty} \frac{m(n)}{\sqrt{n}} \int_{-\infty}^{\infty} d y \int_{4}^{\infty} d \tilde{s} \frac{e^{-\sqrt{n\left(\tilde{s}+y^{2}\right)} \lambda_{\pi}}}{\tilde{s}+2 \mathrm{i} y} \operatorname{disc}\left[\mathcal{F}_{\pi}(\tilde{s}, 1+\mathrm{i} y)\right] .
\end{aligned}
$$

with $m(n) \equiv$ number of integer vectors $\mathbf{z}$ with $\mathbf{z}^{2}=n . I_{p}$ denotes the pole and $I_{c}$ the cut contribution, whose meaning and precise definitions will be explained below. The expression for $\Sigma^{(2)}$ is more cumbersome:

$$
\Sigma^{(2)}=M_{\pi}^{2} \xi^{2}\left[\frac{9}{8} \tilde{g}_{1}\left(\lambda_{\pi}\right)^{2}-\frac{1}{8} \lambda_{\pi} \tilde{g}_{1}\left(\lambda_{\pi}\right) \frac{\partial}{\partial \lambda_{\pi}} \tilde{g}_{1}\left(\lambda_{\pi}\right)+\Delta\right]+\mathcal{O}\left(\xi^{3}\right)
$$

and we merely note that it can be split into products of one-loop contributions (terms with $\tilde{g}_{1}^{2}$ and a pure two-loop part, denoted by $\Delta$. We have introduced the abbreviation

$$
\xi=\frac{M_{\pi}^{2}}{16 \pi^{2} F_{\pi}^{2}}
$$

A detailed derivation of these results will be given in the subsequent sections and we confine ourselves to a few comments at this stage: In $I_{p}$ one recovers the asymptotic formula of Lüscher, if one restricts the sum to the first addendum. Its extension to the present form has already been suggested in [11] and was applied in [12. The function $\mathcal{F}_{\pi}(\nu)$ denotes the isospin zero $\pi \pi$ scattering amplitude in the forward kinematics. It contains cuts due to the two-pion intermediate state. In the derivation of the asymptotic formula, Lüscher consistently dropped contributions arising from the cuts, since in a large volume expansion they are beyond the order of accuracy he aimed at. Here, we take them into account up to two-loops, relying on the chiral representation of $\pi \pi$ scattering amplitude. The outcome of the analysis is summarized in $I_{c}$ in which the same symbol $\mathcal{F}_{\pi}$, this time with two arguments appears: with the latter we mean the scattering amplitude of two on-shell pions into two off-shell pions in the forward kinematics configuration. The contributions 
from the cuts can still be written as integrals over the $\pi \pi$ scattering amplitude with an exponential weight, as in the asymptotic formula. Notice however, that this formula explicitly relies on the chiral representation of the scattering amplitude and is only valid up to two-loops, whereas the Lüscher formula holds to all orders.

Contributions from two pion propagators in finite volume are ultimately captured in $\Sigma^{(2)}$, being expressed in terms of a dimensionless function $\tilde{g}_{1}\left(\lambda_{\pi}\right)$ and a numerically small correction $\Delta$ arising from graph $1 \mathrm{~d}$ ). Both are explicitly given in app. A and eq. (44), respectively.

The pure gauge contributions are not volume dependent and merely renormalize the pion mass, cf. eq. (19). A detailed discussion of this calculation can be found in [33, 9], with which we agree - this was a useful, nontrivial check for our calculation.

\subsection{Self-energy to first order: $\Sigma^{(1)}$}

The simple fields can be summed up in closed form and may be represented by a skeleton diagram, see fig. $2 a$ )

$$
\Sigma^{(1)}=\frac{1}{2} \int \frac{d^{4} q}{(2 \pi)^{4}} \sum_{n=1}^{\infty} m(n) e^{i q_{1} \sqrt{n} L} G_{0}\left(q^{2}\right) \Gamma_{\pi \pi}(\hat{p}, q,-\hat{p},-q),
$$

with $\hat{p}=\left(i M_{\pi}, \mathbf{0}\right)$ and $\Gamma_{\pi \pi}(\hat{p}, q,-\hat{p},-q)$ the 4 -point function of $\pi \pi$ scattering in the forward scattering kinematics. Note that the 4-point function in eq. (25) is an off-shell amplitude and that we evaluate it at $\hat{p}$ and not at $\hat{p}_{L}$. The difference is taken into account in $\Sigma^{(2)}$. The representation of $\Sigma^{(1)}$ in eq. (25) has already been used by Lüscher and eventually led him to the asymptotic formula [1]. He then discussed the contribution of the pole of the propagator $G_{0}\left(q^{2}\right)$ that one meets at

$$
q_{1}=i \sqrt{M_{\pi}^{2}+q_{\perp}^{2}+q_{0}^{2}}, \quad q_{\perp}=\left(q_{2}, q_{3}\right) .
$$

Above this pole the singularities come from the cuts of the propagator and the $4-$ point function $\Gamma_{\pi \pi}(\hat{p}, q,-\hat{p},-q)$. These start from

$$
s=-(\hat{p}+q)^{2} \geq 4 M_{\pi}^{2}, \quad u=-(\hat{p}-q)^{2} \geq 4 M_{\pi}^{2}, \quad-q^{2} \geq 9 M_{\pi}^{2} .
$$

Lüscher showed that the pole contribution is dominating with respect to those coming from the cuts and neglected the latter. In fact, his discussion involved no further assumptions about the 4-point function and remains true at every order of the perturbative expansion. Since our goal is to test the asymptotic formula beyond the leading exponentials, we wish to work out the impact of the contributions that were dropped by Lüscher. Doing this at the two-loop level is rather straightforward, as we will now show. 


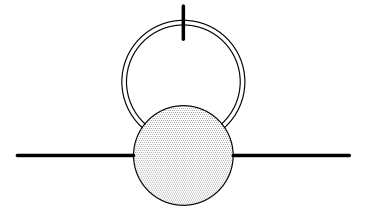

a)

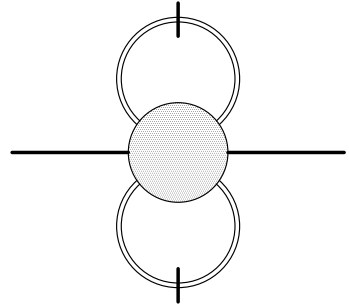

b)

Figure 2: Skeleton diagrams representing $a$ ) eq. (25) and b) eq. (64). The blob in a) stands for the 4-point function of $\pi \pi$ scattering in infinite volume and in $b$ ) for a subtracted 6 -point function of $\pi \pi \pi$ scattering in infinite volume. The double-line with the spline is a finite volume propagator with the physical pion mass $M_{\pi}^{2}$.

Up to $\mathcal{O}\left(p^{4}\right)$, the four-point function can be decomposed into a combination of functions which have either a singularity in $s$ or in $u$. Since $\Sigma^{(1)}$ is symmetric in $s$ and $u$, we may write $\Gamma_{\pi \pi}(\hat{p}, q,-\hat{p},-q)$ in terms of a function $\bar{\Gamma}_{\pi \pi}(s, \hat{p} q)$, which depends only on the variables $s$ and $\hat{p} q$, and which has cuts at $s \geq 4 M_{\pi}^{2}$,

$$
\Gamma_{\pi \pi}(\hat{p}, q,-\hat{p},-q)=\bar{\Gamma}_{\pi \pi}(s, \hat{p} q)+\mathcal{O}\left(\frac{1}{F_{\pi}^{6}}\right)
$$

We write $q_{1}=x+i y$ and for the domain $s \in \mathbb{R}, s \geq 4 M_{\pi}^{2}$ we have

$$
y^{2}-x^{2}-q_{0}^{2}-q_{\perp}^{2} \geq 3 M_{\pi}^{2}, \quad x y=-q_{0} M_{\pi} .
$$

In particular we observe that in the complex $q_{1}$ plane the cut starts above the pole of eq. (26), as illustrated in fig. 3. Performing now the contour integration in the upper half plane we obtain two terms: the first one, $I_{p}$, comes from the pole and is simply its residuum. The other comes from the integral along the new integration path. The latter contribution vanishes as we push the integration lines to infinity, except for the path which goes around the cut, to be denoted by $I_{c}$ in the following,

$$
\Sigma^{(1)}=I_{p}+I_{c}
$$

As was shown by Lüscher [1], the former can be simplified considerably and can be brought in the usual form of the asymptotic formula

$$
I_{p}=\frac{M_{\pi}^{2}}{16 \pi^{2} \lambda_{\pi}} \sum_{n=1}^{\infty} \frac{m(n)}{\sqrt{n}} \int_{-\infty}^{\infty} d y \mathcal{F}_{\pi}(\mathrm{i} y) e^{-\sqrt{n\left(1+y^{2}\right)} \lambda_{\pi}}
$$




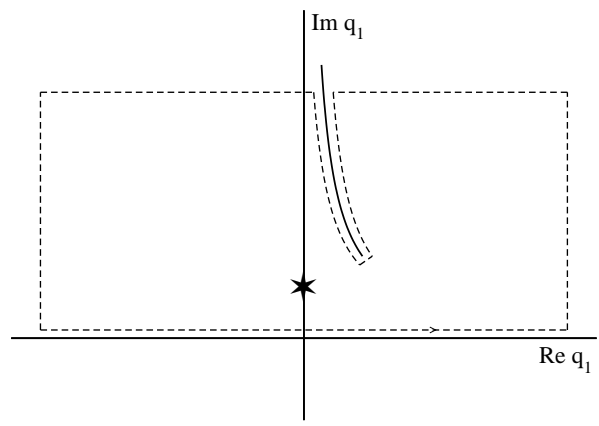

Figure 3: Integration contour in the complex $q_{1}$ plane with the pole from the pion propagator and the branch cut from the $\pi \pi$ scattering amplitude.

with $\mathcal{F}_{\pi}(i y)$ the $\pi \pi$ forward scattering amplitude. Restricting the sum to the first term we recover Lüscher's formula [1]. For $I_{c}$ we find

$$
I_{c}=\frac{1}{2} \sum_{n=1}^{\infty} m(n) \int \frac{d q_{0} d^{2} q_{\perp}}{(2 \pi)^{4}} \int_{\left[s \geq 4 M_{\pi}^{2}\right]} d q_{1} \frac{e^{i q_{1} \sqrt{n} L}}{M_{\pi}^{2}+q^{2}} \operatorname{disc}\left[\bar{\Gamma}_{\pi \pi}(s, \hat{p} q)\right]+\mathcal{O}\left(F_{\pi}^{-6}\right),
$$

where $\operatorname{disc}\left[\bar{\Gamma}_{\pi \pi}(s, \hat{p} q)\right]$ denotes the discontinuity of $\bar{\Gamma}_{\pi \pi}$ along the cut. It is now convenient to shift the integration path in $q_{0}$ from $\operatorname{Im}\left(q_{0}\right)=0$ to $\operatorname{Im}\left(q_{0}\right)=-\mathrm{i} M_{\pi}$. Along this path we have

$$
q_{0}=\bar{q}_{0}-\mathrm{i} M_{\pi}, \quad s=-\bar{q}_{0}^{2}-q_{1}^{2}-q_{\perp}^{2}, \quad \bar{q}_{0} \in \mathbb{R},
$$

and the integration over $q_{1}=x+\mathrm{i} y$ falls onto the imaginary axis,

$$
\begin{aligned}
x & =0, \quad y \geq y_{0}=\sqrt{4 M_{\pi}^{2}+\bar{q}_{0}^{2}+q_{\perp}^{2}}, \\
I_{c} & =\frac{\mathrm{i}}{2} \sum_{n=1}^{\infty} m(n) \int \frac{d \bar{q}_{0} d^{2} q_{\perp}}{(2 \pi)^{4}} \int_{y_{0}}^{\infty} d y e^{-y \sqrt{n} L} \frac{\operatorname{disc}\left[\bar{\Gamma}_{\pi \pi}(s, \hat{p} q)\right]}{M_{\pi}^{2}+q^{2}} .
\end{aligned}
$$

Next, we change the integration variable from $q_{1}$ to $s$,

$$
I_{c}=-\frac{\mathrm{i}}{2} \sum_{n=1}^{\infty} m(n) \int \frac{d \bar{q}_{0} d^{2} q_{\perp}}{(2 \pi)^{4}} \int_{4 M_{\pi}^{2}}^{\infty} d s \frac{e^{-\sqrt{n\left(s+\bar{q}_{0}^{2}+q_{\perp}^{2}\right)} L}}{2\left(s+\bar{q}_{0}^{2}+q_{\perp}^{2}\right)^{1 / 2}} \frac{\operatorname{disc}\left[\bar{\Gamma}_{\pi \pi}(s, \hat{p} q)\right]}{s+2 \mathrm{i} M_{\pi} \bar{q}_{0}}
$$

and make use of

$$
\int \frac{d^{2} q_{\perp}}{(2 \pi)^{2}} \frac{1}{2\left(\mu^{2}+q_{\perp}^{2}\right)^{1 / 2}} e^{-\sqrt{n\left(\mu^{2}+q_{\perp}^{2}\right)} L}=\frac{1}{4 \pi \sqrt{n} L} e^{-\mu \sqrt{n} L},
$$

to carry through the integration over $q_{\perp}$ and to end up with eq. (22). 


\subsection{Self-energy to second order: $\Sigma^{(2)}$}

The term $\Sigma^{(2)}$ is more complicated to manipulate, although it is suggestive to think that it may be related to an integral over the $3 \pi \rightarrow 3 \pi$ amplitude. A similar question has been asked in the framework of finite temperature QCD [23]. We discuss the situation in finite volume in appendix $B$. Since these considerations have not lead us to a nice and compact representation for $\Sigma^{(2)}$ we have written it as in (23) where we only have split the factorizable two-loop contributions from the rest. The latter, which we have denoted by $\Delta$ we have evaluated numerically.

We conclude this section with a remark concerning the large $L$ behaviour of the two-loop results. In the large volume limit, the contributions from eq. (21) behave according to

$$
\lim _{L \rightarrow \infty} I_{p} \sim \frac{1}{\lambda_{\pi}^{3 / 2}} e^{-\lambda_{\pi}}
$$

Similarly, we may evaluate the large volume behaviour of the terms occurring in eqs. (2322). It turns out that these, besides being exponentially suppressed and behaving at least as $\exp \left(-\alpha \lambda_{\pi}\right)$, with $\alpha=(\sqrt{3}+1) / \sqrt{2}$, are also suppressed by a power of $\lambda_{\pi}$. Unfortunately this suppression is not particularly strong, being of $1 / \sqrt{\lambda_{\pi}}$ with respect to eq. (35). While we can conclude that the resummed Lüscher formula is dominating in comparison to other two-loop diagrams in a $1 / \sqrt{\lambda_{\pi}}$ expansion, i.e.

$$
\begin{aligned}
& M_{\pi L}=\bar{M}_{\pi L}\left(1+\mathcal{O}\left(e^{-\alpha \lambda_{\pi}} / \sqrt{\lambda_{\pi}}\right)\right) \\
& \bar{M}_{\pi L}=M_{\pi}-\frac{1}{2 M_{\pi}} I_{p},
\end{aligned}
$$

we are convinced that the most important test of the resummed formula is the numerical one, which we will discuss in the following.

\section{Summary of analytical results}

In this section we shall give the analytical results in explicit form, i.e. insert the chiral representation of the amplitudes which appear in the formulae given in the previous section and express our results in terms of a few basic integrals. In eq. 119 24) we have split the finite size effects of the pion mass into $\Sigma^{(1)}=I_{p}+I_{c}$ and $\Sigma^{(2)}$. For the former two we find

$$
I_{p}=M_{\pi}^{2} \sum_{n=1}^{\infty} \frac{m(n)}{\sqrt{n}} \frac{1}{\lambda_{\pi}} \xi\left[I_{M_{\pi}}^{(2)}+\xi I_{M_{\pi}}^{(4)}\right],
$$




$$
I_{c}=M_{\pi}^{2} \sum_{n=1}^{\infty} \frac{m(n)}{\sqrt{n}} \frac{1}{\lambda_{\pi}} \xi^{2} \tilde{I}_{c},
$$

where $\xi$ is the chiral expansion parameter defined in eq.(24) and the expressions $I_{M_{\pi}}^{(2)}, I_{M_{\pi}}^{(4)}$ have already been given in Ref. [6, 12], and we reproduce them here for completeness:

$$
\begin{aligned}
I_{M_{\pi}}^{(2)} & =-B^{0} \\
I_{M_{\pi}}^{(4)} & =B^{0}\left[-\frac{55}{18}+4 \bar{\ell}_{1}+\frac{8}{3} \bar{\ell}_{2}-\frac{5}{2} \bar{\ell}_{3}-2 \bar{\ell}_{4}\right]+B^{2}\left[\frac{112}{9}-\frac{8}{3} \bar{\ell}_{1}-\frac{32}{3} \bar{\ell}_{2}\right] \\
& +\frac{13}{3} R_{0}^{0}-\frac{16}{3} R_{0}^{1}-\frac{40}{3} R_{0}^{2},
\end{aligned}
$$

where

$$
B^{0,2} \equiv B^{0,2}\left(\sqrt{n} \lambda_{\pi}\right), \quad B^{0}(x)=2 K_{1}(x), \quad B^{2}(x)=2 K_{2}(x) / x,
$$

and the integrals $R_{0}^{k}$ are defined as

$$
R_{0}^{k}=\left\{\begin{array} { l } 
{ \operatorname { R e } } \\
{ \operatorname { I m } }
\end{array} \int _ { - \infty } ^ { \infty } d \tilde { y } \tilde { y } ^ { k } e ^ { - \sqrt { n ( 1 + \tilde { y } ^ { 2 } ) } \lambda _ { \pi } } g ( 2 + 2 \mathrm { i } \tilde { y } ) \quad \text { for } \left\{\begin{array}{c}
k \text { even } \\
k \text { odd }
\end{array},\right.\right.
$$

where $g(x)$ is related to the standard one-loop function $\bar{J}\left(x M_{\pi}^{2}\right)$ through

$$
g(x)=16 \pi^{2} \bar{J}\left(x M_{\pi}^{2}\right),
$$

and $\bar{J}\left(x M_{\pi}^{2}\right)$ given in eq. (56). For instance for $x<0$, we have

$$
g(x)=\sigma \log \frac{\sigma-1}{\sigma+1}+2, \quad \text { with } \quad \sigma=\sqrt{1-4 / x},
$$

and elsewhere defined through analytic continuation. In Ref. 12 also the next-tonext-to-leading order term in $I_{p}$, namely $\xi^{2} I_{M_{\pi}}^{(6)}$ has been given and we will use it in our numerical analysis. The coefficient $\tilde{I}_{c}$ can be expressed as a combination of basic integrals

$$
\begin{aligned}
\tilde{I}_{c} & =\frac{1}{3}\left(112 C^{0,2}+37 C^{1,0}-40 C^{1,2}-4 C^{2,0}\right) \\
C^{j, k} & =\int_{-\infty}^{\infty} d y \int_{4}^{\infty} d \tilde{s} \frac{e^{-\sqrt{n\left(\tilde{s}+y^{2}\right)} \lambda_{\pi}}}{\tilde{s}^{2}+4 y^{2}}\left(1-\frac{4}{\tilde{s}}\right)^{1 / 2} \tilde{s}^{j} y^{k} .
\end{aligned}
$$

Notice that the latter expression is obtained from the chiral representation of the off-shell $\pi \pi$ scattering amplitude for forward kinematics. This is parameterization dependent, and the result given here is obtained for the parameterization discussed in sect. 2.1] The dependence on the parameterization must cancel in the full result. 
The self-energy to second order has already been introduced in eq.(23) and reproduced here for convenience

$$
\Sigma^{(2)}=M_{\pi}^{2} \xi^{2}\left[\frac{9}{8} \tilde{g}_{1}\left(\lambda_{\pi}\right)^{2}-\frac{1}{8} \lambda_{\pi} \tilde{g}_{1}\left(\lambda_{\pi}\right) \frac{\partial}{\partial \lambda_{\pi}} \tilde{g}_{1}\left(\lambda_{\pi}\right)+\Delta\right]+\mathcal{O}\left(\xi^{3}\right),
$$

with

$$
\Delta=\left(16 \pi^{2}\right)^{2}\left[4 \tilde{p}_{\mu} \tilde{p}_{\nu} H_{\mu \nu}+4 \tilde{p}_{\mu} H_{\mu}+\frac{7}{6} H\right], \quad \tilde{p}=\frac{\hat{p}}{M_{\pi}},
$$

where $H, H_{\mu}$ and $H_{\mu \nu}$ are related to the sunset-type integrals of fig. 1 l l). We have not been able to find a compact representation for these integrals and only elaborate on their numerical analysis in app. $\mathrm{A}$ in some detail.

\section{$5 \quad$ Numerics}

\subsection{Setup}

The numerical analysis is performed in line with the setup of Ref. 12]. The quantity of interest is

$$
R_{M_{\pi}} \equiv \frac{M_{\pi L}-M_{\pi}}{M_{\pi}}
$$

whose quark mass dependence shall be evaluated numerically for different sizes $L$. The parameters of $R_{M_{\pi}}$ are (see eqns. (19) 24) and eqns.(37 444) the pion mass $M_{\pi}$

and the pion decay constant $F_{\pi}$ in infinite volume as well as (implicitly in $I_{M_{\pi}}^{(4 / 6)}$ ) the $\mathrm{SU}(2)$ low energy constants. The quark mass dependence of the pion decay constant may be taken into account by expressing $F_{\pi}$ as a function of the pion mass $M_{\pi}$ (see, e.g. [6]). Regarding the low energy constants, we use the ones determined in [9, 10] which are the same as in our previous finite size studies [6, 12].

\subsection{Results}

We plot our results for $R_{M_{\pi}}$ in figs. [4 and 5 both for $L=2,3,4 \mathrm{fm}$ as a function of $M_{\pi}$ and for $M_{\pi}=100,300,500 \mathrm{MeV}$ as a function of $L$. We show the one-loop result (LO) as well as the two-loop result (NLO). These shall be compared with the resummed asymptotic formula with $\mathrm{LO} / \mathrm{NLO} / \mathrm{NNLO}$ input for the $\pi \pi$ scattering amplitude. Note that the one-loop result and the resummed asymptotic formula to LO coincide. The best estimate for $R_{M_{\pi}}$ is finally obtained by adding to the asymptotic pure three-loop contribution the two-loop result (NNLO asympt. + full NLO). At NLO, the finite size effects contain low energy constants, see eg. diagram $f$ ) and $g$ ) in fig. 1, leading to a non-negligible error band. 


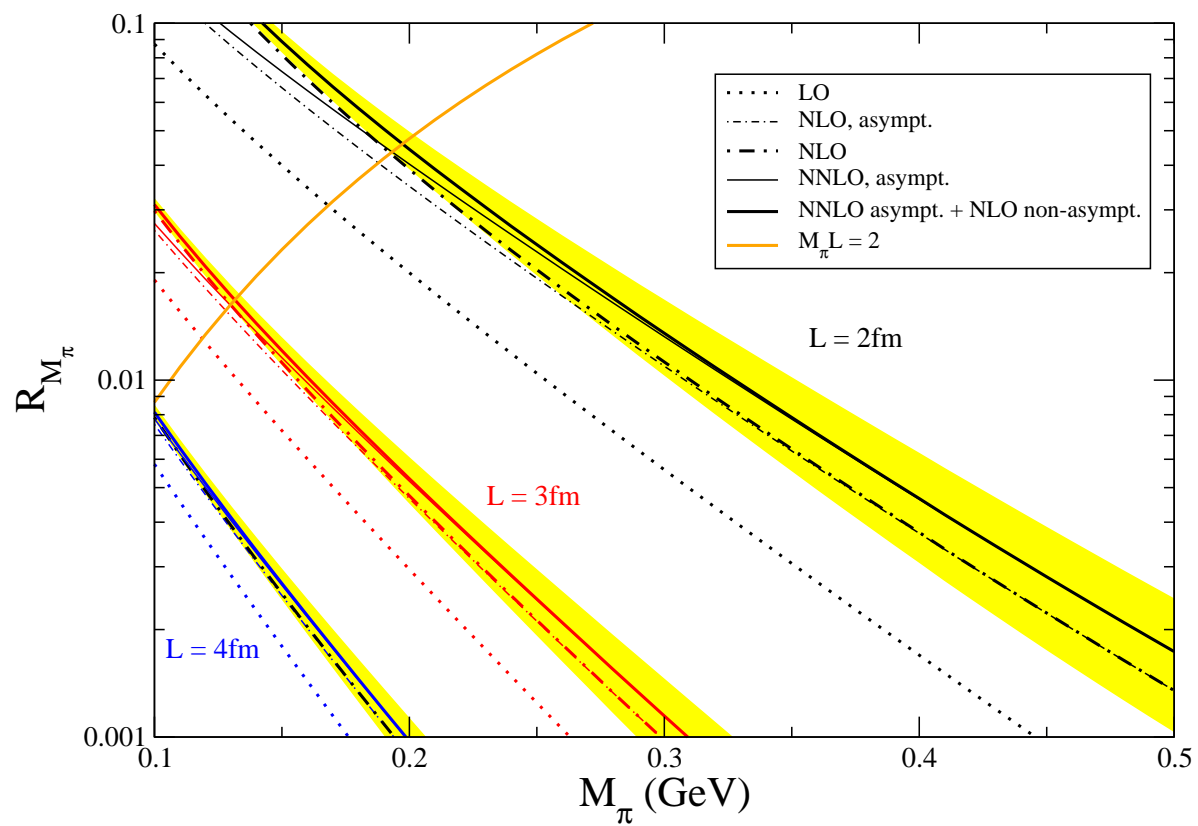

Figure 4: $R_{M_{\pi}}=M_{\pi L} / M_{\pi}-1$ vs. $M_{\pi}$ for $L=2,3,4 \mathrm{fm}$. The result of the resummed asymptotic Lüscher formula (21) with $\mathrm{LO} / \mathrm{NLO} / \mathrm{NNLO}$ chiral input (with attribute "asympt." for NLO and NNLO in legenda) is compared to the one-loop (LO) and two-loop (NLO) result. The best estimate for $R_{M_{\pi}}$ is obtained by adding the pure three loop contribution from the asymptotic formula to the two-loop result (NNLO asympt.+ full NLO). The error band comes from the uncertainties in the low energy constants and is only shown for the best estimate. In the region above the $M_{\pi} L=2$ line, one is not safely in the $p$-regime and our results should not be trusted.

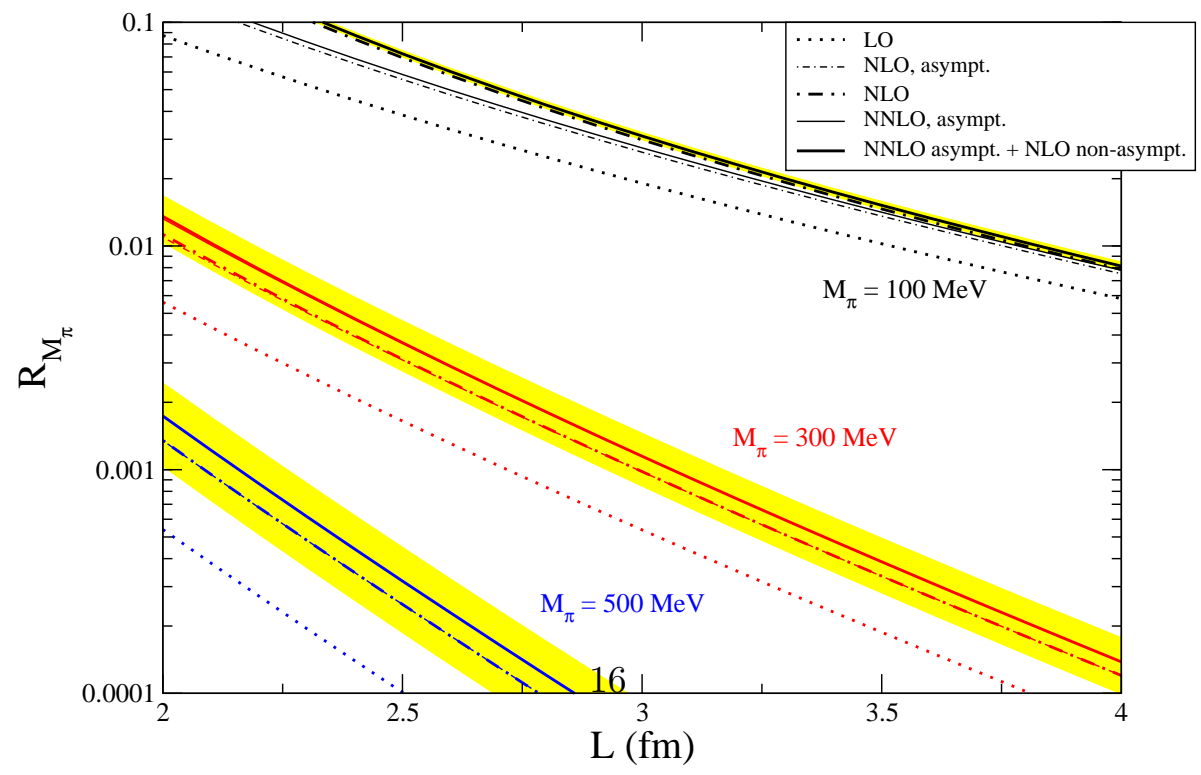

Figure 5: $R_{M_{\pi}}=M_{\pi L} / M_{\pi}-1$ vs. $L$ for $M_{\pi}=100,300,500 \mathrm{MeV}$. The rest of the legenda is as in fig. 4 . 


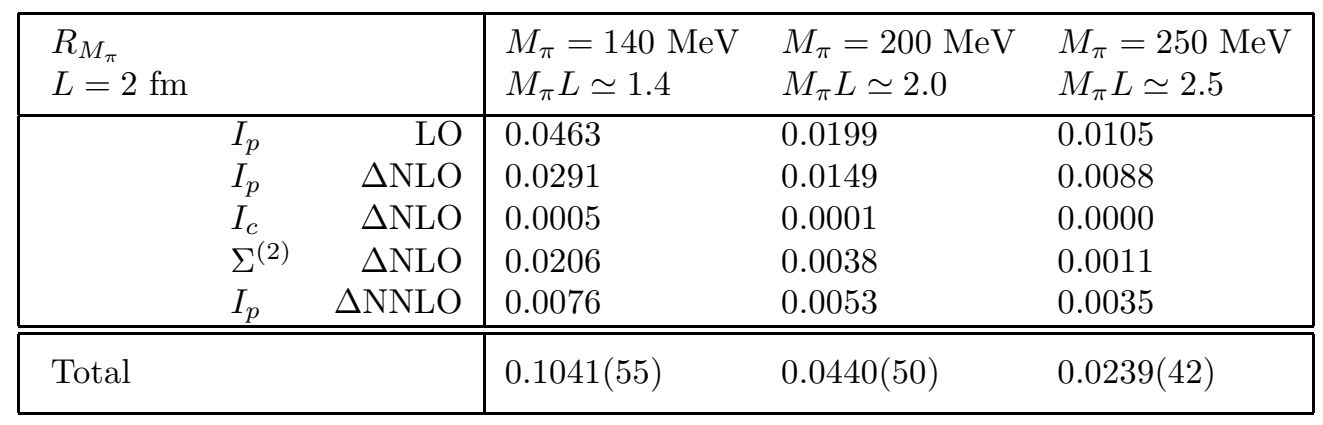

Table 1: $R_{M_{\pi}}=M_{\pi L} / M_{\pi}-1$ for a $2 \mathrm{fm}$ volume and pion masses $M_{\pi}=140 \mathrm{MeV}$, $200 \mathrm{MeV}, 250 \mathrm{MeV}$. We show the relative numerical impact of the LO, the pure NLO and the pure NNLO contribution for $R_{M_{\pi}}$. In the second column we give the source of the effect. The fifth line, e.g. contains the contribution to $R_{M_{\pi}}$ of the dispersive terms $I_{c}$ alone, once kinematical prefactors are added accordingly. The numerical results for the $I_{p}$ contributions, to LO, NLO and NNLO have already been given in [6, 12].

We take up a point which was already observed in [5], namely the large contributions when going from LO to NLO in the asymptotic formula (dotted to thin-dash-dotted). Compared to this gap, the additional contributions from the full two-loop result (thick-dash-dotted) are very small. The two-loop and the NLO result from the asymptotic formula only drift away, when we go beyond the region where the $p$-regime can be safely applied. In tab. 1 we wish to underline these statements with a numerical example: we show the relative numerical impact of the LO, the pure NLO and the pure NNLO contribution for $R_{M_{\pi}}$. In the second column we give the source of the effect. The fifth line, e.g. contains only the contribution of the dispersive terms $I_{c}$ to $R_{M_{\pi}}$, once the kinematical prefactors are properly accounted for. We observe that $I_{c}$ is strongly suppressed, irrespective of the value $M_{\pi} L$. Consider the column with $M_{\pi} L \simeq 1.4$. Although the bulk of the subleading effects is still due to the asymptotic contributions, the terms of $\Sigma^{(2)}$ play a significant role and can not be neglected. This behaviour was expected, since with $M_{\pi} L \simeq 1.4$ we might have already crossed the border of the $p$-regime. As we increase this parameter to $M_{\pi} L \simeq 2$, the asymptotic regime begins to set in. The contributions from the resummed asymptotic formula are now dominating with respect to those from $\Sigma^{(2)}$. The numerical results for $M_{\pi} L \simeq 2.5$ confirm this trend. The fact that even the additional NNLO asymptotic terms (a partial three-loop result) are larger than the NLO non-asymptotic contributions is in nice agreement with the analytical expectation found in eq. (36). However, we 
find that the suppression seen in the numbers is stronger than what one could have expected on the basis of the latter analytical argument. Finally, the discussion of the subleading effects allows us to give a reliable estimate for the lower bound of $M_{\pi} L$ for the $p$-regime,

$M_{\pi} L \gtrsim 2: \quad$ lower bound for $p$-regime .

\section{Summary}

i) We have evaluated the finite volume corrections for the pion mass to twoloops within the framework of chiral perturbation theory (ChPT) in the $p$-regime $\left(M_{\pi} L \gg 1, L>2 \mathrm{fm}, M_{\pi}<500 \mathrm{MeV}\right)$.

ii) We have compared the two-loop result with the resummed version of the asymptotic formula of Lüscher. We have found that whenever the effects are calculated for masses and volumes such that $M_{\pi} L \gg 1$, such that one is safely within the p-regime of ChPT, the contributions which are not included in the resummed asymptotic formula are very small. The result gives us confidence in the claim that the resummed asymptotic formula is a convenient and efficient way to reliably calculate finite volume effects for hadronic masses [12.

iii) The derivation of the asymptotic formula for decay constants 34 follows closely the original one for the masses [1] - the only new, subtle point, concerns the amplitude which appears in the integrand of the asymptotic formula, which has a pole in the integration region which first needs to be subtracted (see 34] for details). This is, however, a technical point - the physics of the finite volume corrections for masses and decay constants appears to be rather similar. In view of this we believe that the present results speak also in favour of the resummed asymptotic formula for decay constants 12]. A check of this claim could be obtained by evaluating the pion decay constant to two loops.

iv) The two-loop calculation performed here allows us to better estimate the region of validity of $\mathrm{ChPT}$ in the $p$-regime. Our conclusion is that in the case of the pion mass it is necessary to have $M_{\pi} L \gtrsim 2$. Again, this serves as a guideline also for decay constants and masses of other hadrons. 


\section{Acknowledgments}

We thank Stephan Dürr for useful discussions and a careful reading of the manuscript. This work has been supported by the Schweizerischer Nationalfonds and partly by the EU "Euridice" program under code HPRN-CT2002-00311.

\section{A Finite volume integrals}

In this appendix we give further details on the finite volume integrals which occurred in the two-loop calculation. Throughout we applied dimensional regularization, as is common in ChPT. Since the finite box breaks Lorentz invariance, tensor simplifications have to be performed with care. Consider e.g. the integral

$$
\hat{p}_{\mu} \hat{p}_{\nu} A_{\mu \nu}=\int \frac{d^{d} q}{(2 \pi)^{d}} \sum_{\mathbf{n} \in \mathbb{Z}^{3}}{ }^{\prime} \frac{e^{\mathrm{iqn} L}}{M_{\pi}^{2}+q^{2}}(\hat{p} q)^{2}
$$

which arises when evaluating diagram $f)$ in fig. 1 Remember $\hat{p}=\left(\mathrm{i} M_{\pi}, \mathbf{0}\right)$. The prime in the sum denotes that the term with $\mathbf{n}=\mathbf{0}$ is excluded. The ansatz

$$
A_{\mu \nu}=\delta_{\mu \nu} A
$$

with $A$ a scalar integral which is valid in infinite volume, leads to an incorrect result in finite volume. A direct calculation of the integral with the help of eq. (61) yields

$$
\hat{p}_{\mu} \hat{p}_{\nu} A_{\mu \nu}=-\frac{\left(M_{\pi}^{2}\right)^{1+d / 2}}{\left(2 \pi \lambda_{\pi}\right)^{d / 2}} \sum_{n=1}^{\infty} \frac{m_{d}(n)}{n^{d / 4}} K_{d / 2}\left(\lambda_{\pi} \sqrt{n}\right) .
$$

In four dimensions the result agrees with the derivation outlined in the next section, where one proceeds along the same lines as in sect. 3.4.

\section{A.1 Tadpole}

In fig. 1. the finite volume corrections of the diagrams $(a)-(c),(f)$ and $(g)$ factorize into one-loop integrals which are of the generic form

$$
\int \frac{d^{4} q}{(2 \pi)^{4}} \sum_{\mathbf{n} \in \mathbb{Z}^{3}} \frac{e^{\mathbf{i q n} L}}{\left(M_{\pi}^{2}+q^{2}\right)^{k}} \mathcal{P}\left(\hat{p} q ; q^{2}\right),
$$

with $k$ an integer positive number and $\mathcal{P}\left(\hat{p} q, q^{2}\right)$ a polynomial of $\hat{p} q$ and $q^{2}$. It suffices to discuss the case for $k=1$, since $k=2,3, \ldots$ are obtained through appropriate derivatives with respect to $M_{\pi}^{2}$. The only singularity of the integrand 
is the pole of the propagator. Therefore, the contour integration analysis applied in sect. 3.4 yields the result

$$
\sum_{n=1}^{\infty} \frac{m(n)}{\sqrt{n}} \frac{M_{\pi}^{2}}{8 \pi^{2} \lambda_{\pi}} \int_{-\infty}^{\infty} d y e^{-\sqrt{n\left(1+y^{2}\right)} \lambda_{\pi}} \mathcal{P}\left(\mathrm{i} M_{\pi}^{2} y ;-M_{\pi}^{2}\right)
$$

In particular, in the text we have used the dimensionless function $\tilde{g}_{1}\left(\lambda_{\pi}\right)$,

$$
\begin{aligned}
\tilde{g}_{1}\left(\lambda_{\pi}\right) & =\frac{16 \pi^{2}}{M_{\pi}^{2}} g_{1}\left(M_{\pi}^{2}, 0, L\right), \\
g_{k}\left(M_{\pi}^{2}, 0, L\right) & =\sum_{\mathbf{n} \in \mathbb{Z}^{3}} / \int \frac{d^{d} q}{(2 \pi)^{d}} \frac{e^{\mathbf{i} \mathbf{q n} L}}{\left(M_{\pi}^{2}+q^{2}\right)^{k}},
\end{aligned}
$$

evaluated at $d=4$, and where $g_{1}\left(M_{\pi}^{2}, 0, L\right)$ was introduced a long time ago by Gasser and Leutwyler [2, 3], see also eq. (14).

\section{A.2 Sunset}

The only real two-loop diagram is the sunset graph fig. 1 1 ), and we will comment on it in some detail. It is convenient to split the finite volume integrals as in eq. (18) only after the tensor simplifications. As alluded in the beginning of the appendix, tensor simplifications in finite volume have to be performed with care. Even though one can not rely on Lorentz invariance, the sunset tensor integrals may still be reduced to the structures

$$
\left\{\mathcal{H}, \mathcal{H}_{\mu}, \mathcal{H}_{\mu \nu}\right\}=\int d^{d} x e^{i p x} G(x)^{2}\left[\left\{1, \mathrm{i} \partial_{\mu},-\partial_{\mu} \partial_{\nu}\right\} G(x)\right],
$$

with $G(x)$ from eq. (77). A rather direct way to perform these steps is to work in coordinate space and to make use of partial integrations, i.e.

$$
\begin{aligned}
\int d^{d} x e^{i p x} \partial_{\mu} G(x) \partial_{\nu} G(x) G(x) & = \\
& -\frac{1}{2} \int d^{d} x e^{i p x} G(x)^{2}\left[p_{\mu} i \partial_{\nu} G(x)+\partial_{\mu} \partial_{\nu} G(x)\right] .
\end{aligned}
$$

Notice that the same identities in momentum space are derived with the help of translational invariance which is still respected in finite volume due to the periodic boundary conditions. In the following, we will only elaborate on the scalar integral $\mathcal{H}$ in more detail. We expand the integral in terms of number of finite volume propagators as motivated in sect. 3.3 .

$$
\mathcal{H}=\mathcal{H}^{(0)}+3 \mathcal{H}^{(1)}+\mathcal{H}^{(2)},
$$


where the first (second) addend corresponds to the pure (simple) gauge fields contribution. For $\mathcal{H}^{(0)}$ we refer to 31]. Further,

$$
\mathcal{H}^{(1)}=\sum_{n=1}^{\infty} m(n) \int \frac{d^{4} q}{(2 \pi)^{4}} \frac{e^{i q_{1} \sqrt{n} L}}{M_{\pi}^{2}+q^{2}} \bar{J}\left[(\hat{p}-q)^{2}\right]+g_{1}\left(M_{\pi}^{2}, 0, L\right) J(0),
$$

with

$$
J\left(k^{2}\right)=\int \frac{d^{d} \ell}{(2 \pi)^{d}} \frac{1}{\left[M_{\pi}^{2}+\ell^{2}\right]} \frac{1}{\left[M_{\pi}^{2}+(k-\ell)^{2}\right]}=\bar{J}\left(k^{2}\right)+J(0) .
$$

The first term of eq. (56) is finite, the second carries an uv-divergence which is absorbed by a counterterm. This shows that although finite volume effects do not generate new uv-divergences, they still appear at intermediate steps of the calculation. It is a thorough check on our calculation that these non-analytic divergences cancel. Finally,

$$
\mathcal{H}^{(2)}=\sum_{\substack{\mathbf{n}, \mathbf{r} \in \mathbb{Z}^{3} \backslash \mathbf{0} \\ \mathbf{n} \neq \mathbf{r}}} \int \frac{d^{4} q}{(2 \pi)^{4}} \frac{d^{4} k}{(2 \pi)^{4}} \frac{e^{i \mathbf{q n} L}}{\left[M_{\pi}^{2}+q^{2}\right]} \frac{e^{i \mathbf{k r} L}}{\left[M_{\pi}^{2}+k^{2}\right]} \frac{1}{\left[M_{\pi}^{2}+(\hat{p}-q-k)^{2}\right]}
$$

In the text, we used its dimensionless version

$$
\left\{H ; H_{\mu} ; H_{\mu \nu}\right\}=\sum_{\substack{\mathbf{n}, \mathbf{r} \in \mathbb{Z}^{3} \backslash \mathbf{n} \\ \mathbf{n} \neq \mathbf{r}}} \int \frac{d^{4} \tilde{q}}{(2 \pi)^{4}} \frac{d^{4} \tilde{k}}{(2 \pi)^{4}} \frac{e^{i \tilde{\mathbf{q}} \mathbf{n} \lambda_{\pi}}}{\left[1+\tilde{q}^{2}\right]} \frac{e^{i \tilde{\mathbf{k}} \mathbf{r} \lambda_{\pi}}}{\left[1+\tilde{k}^{2}\right]} \frac{\left\{1 ; \tilde{k}_{\mu} ; \tilde{k}_{\mu} \tilde{k}_{\nu}\right\}}{\left[1+(\tilde{p}-\tilde{q}-\tilde{k})^{2}\right]},
$$

which are uv-finite and do not need to be renormalized. We only have to find a convenient representation for the numerical analysis. We shall restrict ourselves to the scalar integral in the following. Introducing a Feynman parameter by combining the second and third denominator, we find

$$
H=\int \frac{d^{4} \tilde{q}}{(2 \pi)^{4}} \frac{d^{4} \tilde{k}}{(2 \pi)^{4}} \int_{0}^{1} d x \sum_{\substack{\mathbf{n}, \mathbf{r} \in \mathbb{Z}^{3} \backslash \mathbf{n} \\ \mathbf{n} \neq \mathbf{r}}} \frac{1}{\left[1+\tilde{q}^{2}\right]} \frac{e^{\mathrm{i} \tilde{\mathbf{q}}(\mathbf{r}-\mathbf{n} x) \lambda_{\pi}+\mathbf{i n} \tilde{\mathbf{k}} \lambda_{\pi}}}{\left[1+(\tilde{p}-\tilde{q})^{2} x(1-x)+\tilde{k}^{2}\right]^{2}}
$$

We use Schwinger's trick for both remaining denominators

$$
\frac{1}{1+x^{2}}=\int_{0}^{\infty} d \alpha e^{-\alpha\left(1+x^{2}\right)}
$$

The integrals over $\tilde{k}$ and $\tilde{q}$ are then of the Gaussian type and can be performed analytically. We are then left with three integrations over a rather lengthy expression which shall not be written down here. Despite their unhandy form, the 
integrations may still safely be performed numerically. The accuracy of the determination of eq. (60) is not limited by the integration routine, but by the rather slow convergence of the sum in $\mathbf{n}$ and $\mathbf{r}$ for moderate $\lambda_{\pi}$. Consequently, the evaluation of the sunset integrals going into $\Delta$ is restricted to three significant digits. (The last digit given for $\Sigma^{(2)}$ in tab. 1 is not significant.) Note that the uncertainty of the $H$-type integrals is not a serious matter. Firstly, it could be lowered by brute force and secondly it only plays a (minor) role, in case when the $p$-regime can not be safely applied anymore.

\section{B Self-energy to second order: $\Sigma^{(2)}$}

In this section we ask ourselves whether the self-energy to second order can be represented in a similar compact form as in the case of the self-energy to first order in eq. (25). As will be discussed, it is indeed possible to relate the selfenergy to second order to a $3 \pi$ scattering amplitude in the forward scattering kinematics, as illustrated in fig. 2b). This has already been observed by Schenk in a related context [23]. He investigated the dynamics of pions in a cold heat bath of temperature $T$ and examined the effective mass of the pion $M_{\pi}(T)$ within the framework of ChPT. The close relation between finite temperature field theory and finite volume effects becomes apparent in the imaginary time formalism, where one treats the inverse temperature as a finite extension in the imaginary time direction. The expansion in terms of the number of finite volume propagators is then in one-to-one correspondence with the expansion in terms of the number of finite temperature propagators. In the following, Schenk's approach shall be adapted to the finite volume scenario. We first establish the relation between $\Sigma^{(2)}$ and the three-to-three particle scattering amplitude and proceed with various remarks.

Consider the 6 -point function in $d$ dimensions in the non-linear sigma model parameterization eq. (3) in the forward kinematics

$$
\begin{aligned}
& \sum_{a, b=1}^{3} \int d x_{1} \ldots d x_{5} e^{-\mathrm{i} p\left(x_{1}-x_{4}\right)-\mathrm{i} k\left(x_{2}-x_{5}\right)-\mathrm{i} q\left(x_{3}-x_{6}\right)}\left\langle 0\left|\mathrm{~T} \varphi_{\mathrm{x}_{1}}^{1} \varphi_{\mathrm{x}_{2}}^{\mathrm{a}} \varphi_{\mathrm{x}_{3}}^{\mathrm{b}} \varphi_{\mathrm{x}_{4}}^{1} \varphi_{\mathrm{x}_{5}}^{\mathrm{a}} \varphi_{\mathrm{x}_{6}}^{\mathrm{b}}\right| 0\right\rangle \\
& \quad=\frac{Z^{3}}{\left(M_{\pi}^{2}+p^{2}\right)^{2}\left(M_{\pi}^{2}+k^{2}\right)^{2}\left(M_{\pi}^{2}+q^{2}\right)^{2}} T_{\pi \pi \pi}(p, k, q)
\end{aligned}
$$

with $Z$ the wave function renormalization constant and $\varphi_{x} \equiv \varphi(x)$. The amplitude $T_{\pi \pi \pi}(p, k, q)$ contains a pole at $\hat{p}^{2}=-M_{\pi}^{2}$ which needs to be subtracted

$$
T_{\pi \pi \pi}(p, k, q)=\hat{T}_{\pi \pi \pi}(p, k, q)+\frac{R(p, k, q)}{M_{\pi}^{2}+p^{2}}
$$




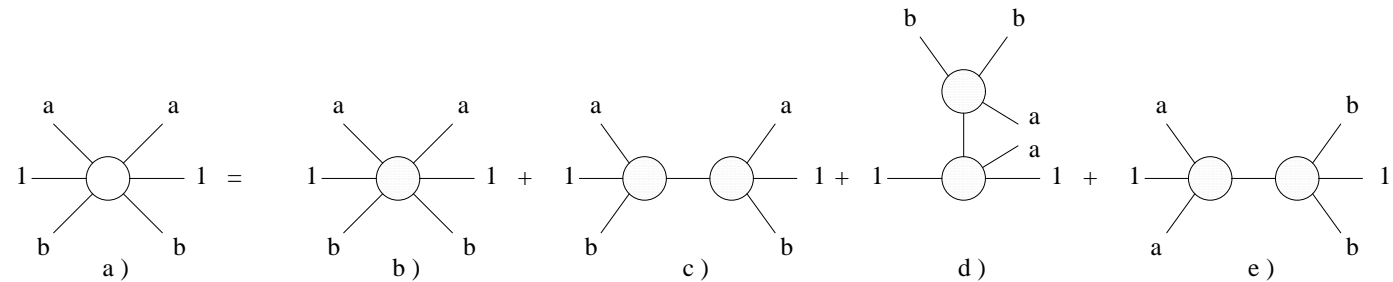

Figure 6: Decomposition of the $3 \pi-3 \pi$ amplitude in terms of 1particle irreducible parts. The characters $1, a$ and $b$ on the external legs denote isospin indices.

The self-energy to second order can then be written in terms of the subtracted amplitude $\hat{T}_{\pi \pi \pi}(\hat{p}, k, q)$

$$
\Sigma^{(2)}=\frac{1}{8} \sum_{\mathbf{n}, \mathbf{r}}^{\prime} \int \frac{d^{4} q}{(2 \pi)^{4}} \frac{d^{4} k}{(2 \pi)^{4}} e^{\mathrm{i} \mathbf{q n} L+\mathrm{i} \mathbf{k r} L} \frac{\hat{T}_{\pi \pi \pi}(\hat{p}, k, q)}{\left(M_{\pi}^{2}+q^{2}\right)\left(M_{\pi}^{2}+k^{2}\right)}+\mathcal{O}\left(\frac{1}{F_{\pi}^{6}}\right)
$$

where the prime restricts the sum to integer vectors $\mathbf{n}$ and $\mathbf{r}$ obeying $\mathbf{n} \neq 0 \neq \mathbf{r}$, and for diagram $c$ ) in fig. [ in addition $\mathbf{n} \neq \mathbf{r}$. The latter restriction avoids double counting as this term is already accounted for in a simple gauge field of $\Sigma^{(1)}$.

i) The reason for the subtraction is easily accounted for. In fig. [6 we decompose the $3 \pi-3 \pi$ scattering amplitude in terms of 1 -particle irreducible parts. The subtraction removes the contribution from the diagram in fig. 6r) which does not correspond to a 1-particle irreducible self-energy diagram, once the external legs are appropriately closed.

A thorough discussion on the physical interpretation of the pole term can be found in [23].

ii) Notice that eq. (63) defines a subtracted off-shell amplitude which depends on the regularization scheme as well as on the parameterization of the pion fields. At the order we are working, the regularization dependence is not an issue, since the subtracted amplitude $\hat{T}_{\pi \pi \pi}(\hat{p}, k, q)$ is only needed at tree level. However, the dependence on the parameterization of the pion fields is of concern. While the momentum integrations in eq. (64) for the diagrams fig. 6b) and 6 l l put the momenta $k$ and $q$ on-shell and the ambiguity due to the parameterization therefore drops out, this does not happen in the case of diagram 6r).

Note that the same parameterization ambiguity already occurred in $I_{c}$ in the dispersive analysis of $\Sigma^{(1)}$ (cf. eq. (31) where $q^{2} \neq-M_{\pi}^{2}$ ). In order to 
understand the close relation between these two terms, we first note that only diagram fig. 1 $(d)$ contributed to $I_{c}$. Further, the simple gauge field of fig. 近 $(d)$ with $\mathbf{n} \neq \mathbf{0}$ for one propagator can immediately be written as a contribution with two finite volume propagators: one periodifies a second propagator, however with the same $\mathbf{n} \neq \mathbf{0}$ as already for the first one. Since $M_{\pi L}$ does not depend on the parameterization of the pion fields, the ambiguities of the two terms have to cancel each other. To explicitly show this is however nontrivial.

In summary, while our representations for $I_{c}$ in eq. (22), resp. eq. (37) and for $\Sigma^{(2)}$ in eq. (64), resp. eq. (23) do depend on the off-shell dependence of the scattering amplitudes, the sum in eq. (19) does not.

iii) Even though the self-energy to second order can be expressed in a compact form, further simplifications (similar to those performed in sect. 3.4 seem not to be straightforward. Instead, for the (numerical) integrations we had to discuss the various terms contributing to $\Sigma^{(2)}$ one by one. A general discussion of finite volume integrals has been provided in app. A. After treating the finite volume integrals, we end up with the basic functions given in eq. (23). 


\section{References}

[1] M. Luscher, Commun. Math. Phys. 104, 177 (1986).

[2] J. Gasser and H. Leutwyler, Phys. Lett. B 184, 83 (1987).

[3] J. Gasser and H. Leutwyler, Phys. Lett. B 188, 477 (1987).

[4] J. Gasser and H. Leutwyler, Nucl. Phys. B 307, 763 (1988).

[5] G. Colangelo, S. Durr and R. Sommer, Nucl. Phys. Proc. Suppl. 119, 254 (2003) hep-lat/0209110.

[6] G. Colangelo and S. Durr, Eur. Phys. J. C 33, 543 (2004) hep-lat/0311023.

[7] J. Gasser and H. Leutwyler, Annals Phys. 158, 142 (1984).

[8] J. Bijnens, G. Colangelo, G. Ecker, J. Gasser and M. E. Sainio, Phys. Lett. B 374, 210 (1996) hep-ph/9511397.

[9] J. Bijnens, G. Colangelo, G. Ecker, J. Gasser and M. E. Sainio, Nucl. Phys. B 508, 263 (1997) [Erratum-ibid. B 517, 639 (1998)] hep-ph/9707291.

[10] G. Colangelo, J. Gasser and H. Leutwyler, Nucl. Phys. B 603, 125 (2001) hep-ph/0103088.

[11] G. Colangelo, Nucl. Phys. Proc. Suppl. 140, 120 (2005) hep-lat/0409111.

[12] G. Colangelo, S. Durr and C. Haefeli, Nucl. Phys. B 721 (2005) 136 arXiv:hep-lat/0503014.

[13] C. Haefeli, hep-lat/0509078.

[14] G. Colangelo, A. Fuhrer and C. Haefeli, hep-lat/0512002.

[15] S. R. Sharpe, Phys. Rev. D 46, 3146 (1992) hep-lat/9205020.

[16] D. Becirevic and G. Villadoro, Phys. Rev. D 69, 054010 (2004) hep-lat/0311028.

[17] A. Ali Khan et al. [QCDSF-UKQCD Collaboration], Nucl. Phys. B 689, 175 (2004) hep-lat/0312030.

[18] D. Arndt and C. J. D. Lin, Phys. Rev. D 70, 014503 (2004) hep-lat/0403012.

[19] S. R. Beane, Phys. Rev. D 70, 034507 (2004) hep-lat/0403015. 
[20] S. R. Beane and M. J. Savage, Phys. Rev. D 70, 074029 (2004) hep-ph/0404131.

[21] P. F. Bedaque, I. Sato and A. Walker-Loud, hep-lat/0601033.

[22] J. Bijnens, N. Danielsson, K. Ghorbani and T. Lahde, hep-lat/0509042.

[23] A. Schenk, Phys. Rev. D 47, 5138 (1993).

[24] D. Toublan, Phys. Rev. D 56, 5629 (1997) hep-ph/9706273.

[25] M. Luscher, DESY 83/116 Lecture given at Cargese Summer Inst., Cargese, France, Sep 1-15, 1983

[26] Y. Koma and M. Koma, Nucl. Phys. B 713, 575 (2005) hep-lat/0406034. Y. Koma and M. Koma, hep-lat/0504009.

[27] J. Braun, B. Klein and H. J. Pirner, Phys. Rev. D 71, 014032 (2005) hep-ph/0408116.

[28] B. Borasoy, G. M. von Hippel, H. Krebs and R. Lewis, hep-lat/0509007.

[29] J. Bijnens, G. Colangelo and G. Ecker, JHEP 9902, 020 (1999) hep-ph/9902437.

[30] J. Bijnens, G. Colangelo and G. Ecker, Annals Phys. 280, 100 (2000) hep-ph/9907333.

[31] J. Gasser and M. E. Sainio, Eur. Phys. J. C 6, 297 (1999) hep-ph/9803251.

[32] P. Hasenfratz and H. Leutwyler, Nucl. Phys. B 343, 241 (1990).

[33] U. Burgi, Nucl. Phys. B 479, 392 (1996) hep-ph/9602429.

[34] G. Colangelo and C. Haefeli, Phys. Lett. B 590, 258 (2004) hep-lat/0403025. 